\title{
¿Quién incide en la gobernanza local en Nicaragua? El caso de los comités municipales de la soberanía y seguridad alimentaria y nutricional
}

Marco Vinicio Moncayo Miño*

José Luis Yagüe Blanco**

doi:I0.III44/Javeriana.cdri3-77.qigl

Recibido: 20I5-09-2I Aprobado: 20I6-0I-20 Disponible en línea: 20I6-06-15

Cómo citar este artículo: Moncayo, M. V. M. y Yagüe Blanco, J. L. (2016). ¿Quién incide en la gobernanza local en Nicaragua? El caso de los comités municipales de la soberanía y seguridad alimentaria y nutricional. Cuadernos de Desarrollo Rural, I3(77), ,I-34. http://dx.doi.org/I0.III44/Javeriana.cdri5-77.qigl

* Universidad Politécnica de Madrid, Candidato a Ph. D. por la Escuela Técnica Superior de Ingenieros Agrónomos de la Universidad Politécnica de Madrid. Consultor para la FAO en temas de gobernanza en Mesoamérica, Ecuador. Correo electrónico: marco.moncayo.mino@gmail.com http://orcid.org/0000-0002-2628-6890.

** Ph. D. por la Universidad Politécnica de Madrid. Investigador titular del Grupo de Investigación en Planificación y Gestión Sostenible del Desarrollo Rural (Gesplan), secretario del Comité Científico de la Asociación Española de Dirección e Ingeniería de Proyectos. Correo electrónico: joseluis.yague@upm.es http://orcid.org/0000-0002-775I-8436 


\title{
Resumen
}

Nicaragua promueve desde el 2009 una estructura interinstitucional específica de seguridad alimentaria. De las instancias planteadas en la respectiva ley, solo se han conformado los comités municipales, los cuales se han convertido en la principal arena política local. Mediante el análisis de redes de políticas se analizó el nivel de incidencia de los actores en las políticas locales. Los resultados presentan a los actores gubernamentales con mayor influencia en la priorización y aprobación de las iniciativas. Aunque la toma de decisiones no se descentraliza del todo, estos espacios suplen las debilidades técnicas y económicas de los gobiernos locales y evitan posibles traslapes entre actores.

\section{Palabra clave:}

gobernanza; análisis de redes; seguridad alimentaria

\section{Who has an Influence on Nicaraguan Local Governance? The Case of the Sovereignty Municipal Committees and the Food and Nutrition Safety}

\begin{abstract}
Since 2009, Nicaragua promotes a specific interinstitutional food safety structure. Out of all instances proposed in said law only municipal committees have been created, which have become the main arena of local politics. By means of social networks analysis, we analyzed the impact level of actors on local politics. The results show government actors with greater impact on initiative prioritization and approval. Although decision taken is not fully decentralized, these spaces replace the technical and economic weaknesses of local governments and avoid overlapping between actors.
\end{abstract}

Keywords:

governance; network analysis; food safety 


\section{Introducción}

A partir de la Declaración Universal de los Derechos Humanos, el Pacto Internacional de Derechos Económicos, Sociales y Culturales y la Cumbre Mundial de la Alimentación, se ha ratificado al derecho a la alimentación dentro de los derechos humanos. Estos instrumentos legales reconocen que toda persona tiene derecho a un nivel de vida adecuado que le asegure la alimentación, y que este derecho debe ser alcanzado progresivamente, siendo los Estados los principales responsables de adoptar las medidas necesarias para erradicar el hambre. Además, han establecido que la condición básica para alcanzar el derecho a la alimentación es la Seguridad Alimentaria y Nutricional (SAN), la cual se "logra cuando todas las personas, en todo momento, disponen de alimentos adecuados, tienen acceso a ellos y los utilizan satisfactoriamente para llevar una vida sana” (Food and Agriculture Organization [FAO], 1996), y han ratificado como deber de los Estados, de instituciones privadas y de la sociedad civil en general, promover instrumentos y mecanismos para garantizar la alimentación adecuada de la población.

Desde el 2006 en Latinoamérica se está buscando garantizar la SAN a través de nuevos marcos legislativos. En esta región son siete países los que han aprobado leyes de seguridad alimentaria y nutricional, y otros diez debaten sobre proyectos de ley similares. Tomando en cuenta los países que ejecutan dichas leyes, Nicaragua presenta la reducción más significativa en los porcentajes de subalimentación: en I990 el 54,4\% de su población se encontraba subalimentada, en el 2015 se ha disminuido hasta I6,6\% (FAO, 20I5). Son muchos los esfuerzos realizados que han permitido alcanzar estos resultados, entre estos se encuentra la puesta en marcha de la Ley no 693 de Soberanía y Seguridad Alimentaria y Nutricional (LSSAN), aprobada en el 2009.

Esta ley impulsa una nueva estructura institucional que busca potenciar el rol del Gobierno en el combate del hambre, situándolo como el principal responsable de la generación de política pública. Este modelo de gobernanza intenta generar cambios en la interdependencia entre actores gubernamentales y no gubernamentales, además, pretende abrir espacios dentro del ciclo de política para que los actores públicos compartan responsabilidades, lo que implica un proceso de adaptación de todos los actores, en especial de los gubernamentales, a condiciones de mayor complejidad sociopolítica (Kooiman, 1993; 2003; Pierre y Peters, 2005; Candel, 2014). Estas acciones buscan adoptar un paradigma de generación y aplicación de política pública basado en redes interinstitucionales.

Sin embargo, después de cinco años desde la entrada en vigor de la LSSAN, no se ha logrado conformar todas las instancias de interdependencia propuestas. A nivel municipal, a diferencia del nivel nacional y distrital, se ha logrado conformar estos 
espacios en 55 municipios. Estos espacios, conocidos como Comisiones Municipales de Soberanía y Seguridad Alimentaria y Nutricional (COMUSSAN), reúnen a la sociedad civil, actores privados y públicos para coordinar acciones conjuntas. Entre las principales responsabilidades que la LSSAN otorga a los COMUSSAN está la coordinación del desarrollo, implementación y evaluación de políticas públicas, y la planificación y evaluación de las acciones SAN. Godek (20I4) en sus estudios sobre la institucionalidad que promueve la LSSAN menciona la importancia de los COMUSSAN como vehículos a través de los cuales, los actores municipales pueden hacer oír sus voces en la formulación de políticas. Además, reconoce a estos espacios como la expresión más fuerte del Estado a nivel municipal y considera que son el primer paso para construir la red de actores vinculados a la SAN en una iniciativa desde abajo hacia arriba.

En este sentido, la implementación de la LSSAN se ha convertido en un proceso de aprendizaje institucional, en el que los actores han realizado esfuerzos para romper las barreras tradicionales de su accionar, introduciendo nuevas formas de toma de decisiones y generando procesos más plurales de gobierno. La LSSAN plantea un cambio en el modelo de gobernanza a través de la reorganización del trabajo del sector público, y una mejor articulación de las iniciativas vinculadas a la SAN, con la sociedad civil y el sector privado, para configurar la red política de actores.

\section{I.I. Gobernanza y análisis de redes}

Las iniciativas que se implementan para combatir el hambre han configurado una compleja red de políticas y programas gubernamentales y no gubernamentales que son la base de la gobernanza nacional y mundial (Pérez-Escamilla, 20ı2). Dicha gobernanza está en constante ajuste por la necesidad de poner en marcha adecuados acuerdos formales e informales entre el Estado y otros actores, que permitan asegurar la sostenibilidad de las políticas que se emprenden (Paarlberg, 2002; Grote, 2014). Desde una visión general, la gobernanza implica introducir una nueva reflexión sobre el papel del Estado y su interacción con la sociedad en la toma de decisiones, cambiando normas establecidas y generando nuevos procesos de gobierno (Rhodes, I996; Fisher, 20II; Fontana y Yagüe, 20I2). También busca la consecución de objetivos comunes, a través de la colaboración de varios actores, lo que tiende a difuminar los límites tradicionales entre el accionar público y el privado (Stoker, 1998). Kooiman (1993) sostiene que los sistemas sociopolíticos contemporáneos no tienen un actor capaz de articular a todos los demás y direccionar la acción colectiva. Los procesos de gobernanza buscan articular intereses de varios actores, para gestionar asuntos comunes a través de un sistema descentralizado. Esto 
ocasiona dinámicas de interdependencia entre actores, aunque no necesariamente los actores interdependientes participan en condiciones de igualdad (Aguilar, 2006). La gobernanza es un paradigma de política que consiste en la incorporación de redes de actores no gubernamentales en el diseño, ejecución y evaluación de políticas públicas, promoviendo comunidades políticas mejor organizadas e interinstitucionales (Rhodes, 2007). Es por tanto un campo de acción para el análisis de redes.

El análisis de redes de políticas va más allá de considerar la estructura de relaciones entre actores y el posicionamiento de cada actor en la red, busca además identificar las características del comportamiento, la percepción y las actitudes de los actores y de la red en su conjunto. También trata de describir las relaciones entre actores, extraer pautas relevantes, trazar el flujo de recursos a través de ellas y descubrir los efectos de dichas relaciones sobre individuos conectados o no (Rodríguez, 2005). Más que centrarse en atributos individuales, el estudio de redes contempla las características de las unidades sociales en tanto que surgen de procesos relacionales. Así, busca comprender las propiedades del entorno estructural y cómo este influye o condiciona el comportamiento de los actores.

Con estas bases, en este artículo se pretende aplicar el análisis de redes al modelo de gobernanza de la SAN en Nicaragua, para identificar el comportamiento de los actores que componen el COMUSSAN y reconocer qué actores son los más representativos en la creación y puesta en marcha de políticas públicas a nivel municipal. De esta manera, se busca entender el comportamiento de la red y los factores que influyen en la articulación de su trabajo.

\section{Metodología}

La LSSAN, aprobada en el año 2009, identificó una serie de actores gubernamentales y no gubernamentales como los responsables de garantizar la seguridad alimentaria y nutricional. Según la ley, estos actores deben conformar instancias interinstitucionales desde el nivel nacional hasta el municipal; a cada una de estas instancias se les otorgó responsabilidades específicas para poner en marcha las políticas públicas. Analizando las responsabilidades que deben asumir estas instancias, se reconoce que la priorización y aprobación de las políticas de SAN es una tarea de los actores gubernamentales involucrados en este proceso, mientras que para la formulación, implementación y evaluación de políticas se propone descentralizar estos roles a los niveles departamentales y municipales, donde se da apertura a la participación de actores no gubernamentales (ver Cofre et al., 2015). La LSSAN señala que el objetivo de 
esta propuesta es coordinar, desde los diferentes niveles territoriales, la articulación de acciones públicas y privadas para la implementación de políticas de SAN. Aunque la ley no especifica las fechas para iniciar o finalizar estos procesos, establece que las instancias de articulación deben asumir sus funciones en un plazo máximo de noventa días, desde julio del 2009 que entró en vigencia la LSSAN.

$\mathrm{Al}$ ser las comisiones municipales los únicos espacios que se ha logrado conformar, el presente trabajo se basó en medir la participación de los actores en la red COMUSSAN a través de un cuestionario estandarizado, para alimentar posteriormente el análisis de redes. La variable utilizada fue la participación de los actores en cada una de las cinco fases del ciclo de política pública (Jan y Wegrich, 2006): I) agenda pública, fase que determina la prioridad política. Desde este punto se promueven iniciativas a través del diálogo y la negociación entre diferentes actores; 2) formulación, fase que expresa el problema, identifica objetivos, metas y alternativas factibles para ser transformadas en programas y políticas; 3) aprobación, fase donde se toman decisiones estratégicas para la puesta en marcha de la política; 4) implementación, fase que incluye todos los procesos posteriores a la aprobación de la política y su impacto en campo; y, 5) evaluación, fase que permite conocer y medir el cumplimiento de los objetivos y el alcance de las metas propuestas. Cada actor identificó en qué fases interactúa con otros actores de la red. A las interacciones se les otorgó el valor de i, y a la ausencia de interacción se le asignó el valor de o.

\section{I. Selección de los actores del COMUSSAN y categorización}

Fueron seleccionados actores técnicos y académicos que participan en la implementación de la LSSAN. A nivel nacional fueron entrevistados los directores de dos organizaciones no gubernamentales que trabajan en varios municipios: el técnico nacional de la FAO que apoya a los COMUSSAN y la responsable de la coordinación del Consejo Interuniversitario de la Soberanía y Seguridad Alimentaria (CIUSSAN). Adicionalmente, se aplicó un muestreo de bola de nieve para incluir a los actores a nivel municipal. Fueron entrevistados 36 actores gubernamentales y no gubernamentales de seis COMUSSAN, correspondientes a los municipios de Somotillo, El Jicaral, El Sauce, Santa Rosa del Peñón, Totogalpa y San Juan de Limay. Para el análisis de la información, los actores fueron clasificados según la institución a la que representan y se agruparon en nueve categorías (tabla r): Magfor (Ministerio de Agropecuario y Forestal), Mefcca (Ministerio de Economía Familiar), Mined (Ministerio de Educación), Minsa (Ministerio de Salud), INTA (Instituto Nacional de Tecnología Agropecuaria), CIUSSAN, gobiernos locales, cooperantes (ONG y organismos internacionales) y asociaciones de productores. 
Esta categorización toma en cuenta la conformación del COMUSSAN propuesta en la LSSAN. Aunque los gabinetes de la familia, la comunidad y la vida no son mencionados como integrantes de forma explícita en la LSSAN, se los incluyó dentro de las categorías porque todos los actores reconocieron su participación. De cuarenta cuestionarios (cuatro nacionales y 36 municipales) fueron eliminados tres, debido a falta de información.

Tabla l. Actores que conforman el Comité Municipal de la Soberanía y Seguridad Alimentaria y Nutricional clasificados por categorías según la institución a la que representan

\begin{tabular}{|c|c|c|c|c|c|}
\hline SECTOR & Categoría & AсtOR & SECTOR & Categoría & AстоR \\
\hline \multirow{27}{*}{$\begin{array}{l}\text { Guberna- } \\
\text { mental }\end{array}$} & Magfor & Magfori & \multirow{27}{*}{$\begin{array}{l}\text { No guber- } \\
\text { namental }\end{array}$} & \multirow{5}{*}{$\begin{array}{l}\text { CIUSSAN } \\
\text { (Consejo } \\
\text { Interuniversitario } \\
\text { de la Soberanía y } \\
\text { Seguridad Alimentaria } \\
\text { y Nutricional) } \\
\end{array}$} & \\
\hline & (Ministerio & & & & \\
\hline & $\begin{array}{l}\text { Agropecuario } \\
\text { y Forestal) }\end{array}$ & Magfor2 & & & CIUSSANl \\
\hline & \multirow[b]{2}{*}{ MEFCCA } & MEFCCAl & & & \\
\hline & & MEFCCA2 & & & \\
\hline & \multirow{2}{*}{$\begin{array}{l}\text { (Ministerio de } \\
\text { Economía Familiar) }\end{array}$} & \multirow[b]{2}{*}{ MEFCCA 3} & & \multirow{6}{*}{$\begin{array}{l}\text { Cooperantes } \\
\text { (FAO, Acción contra } \\
\text { el Hambre, World } \\
\text { Vision, Food for the } \\
\text { hungry, Dimensión } \\
\text { del Desarrollo) }\end{array}$} & FAOl \\
\hline & & & & & $\mathrm{FAO} 2$ \\
\hline & \multirow{2}{*}{ Mined } & MinedI & & & $\underline{\mathrm{ACH}}$ \\
\hline & & Mined2 2 & & & wV \\
\hline & \multirow{2}{*}{$\begin{array}{l}\text { (Ministerio de } \\
\text { Educación) }\end{array}$} & \multirow{2}{*}{ Mined; } & & & $\mathrm{FH}$ \\
\hline & & & & & DIDES \\
\hline & \multirow{2}{*}{ Minsa } & Minsai & & \multirow{12}{*}{$\begin{array}{l}\text { Asociación de } \\
\text { productores } \\
\text { (Asociación de } \\
\text { Ganaderos de El } \\
\text { Sauce, Asociación } \\
\text { Campesino a } \\
\text { Campesino-ADECiAC, } \\
\text { Asociación Ciudadana } \\
\text { de Amistad con } \\
\text { Valles Orientales, } \\
\text { Federación Nacional } \\
\text { de Cooperativas, } \\
\text { Asociación Soya } \\
\text { de Nicaragua) }\end{array}$} & AGES \\
\hline & & Minsa2 & & & Adeciac \\
\hline & (Ministerio de Salud) & Minsaz & & & Aciavo \\
\hline & \multirow[b]{2}{*}{ INTA } & INTAl & & & Aciavo2 \\
\hline & & INTA2 & & & Fenacoop \\
\hline & \multirow{2}{*}{$\begin{array}{l}\text { (Instituto Nacional } \\
\text { de Tecnología } \\
\text { Agropecuaria) }\end{array}$} & INTA3 & & & Soynica \\
\hline & & INTA4 & & & \\
\hline & \multirow{9}{*}{$\begin{array}{l}\text { Gobiernos locales } \\
\text { (Gobiernos de los } \\
\text { municipios de El } \\
\text { Sauce, El Jicaral, } \\
\text { San Juan de Limay, } \\
\text { Santa Rosa del Peñón, } \\
\text { Somotillo y Totogalpa) }\end{array}$} & Local g.I & & & \\
\hline & & Local g.2 & & & \\
\hline & & Local g.3 & & & \\
\hline & & Local g.4 & & & \\
\hline & & Local g. 5 & & & \\
\hline & & Local g.6 & & & \\
\hline & & Local g.7 & & & \\
\hline & & Local g.8 & & & \\
\hline & & Local g.9 & & & \\
\hline
\end{tabular}

FuENTE: elaboración propia 


\subsection{Análisis de redes politicas}

Los datos fueron sometidos a análisis de redes a través de Ucinet (Borgatti et al., 2002). Se considera que un actor tiene mayor o menor importancia si sus conexiones hacen que sea particularmente visible en la red (Wasserman y Faust, 1994). Para ello se analizó la importancia de los actores a través de la centralidad en la red, medida por la centralidad por intermediación, la centralidad por cercanía a otros actores y el grado de centralidad.

La importancia estructural de la red se evaluó a través de la centralidad por intermediación, medida esta que permite contrastar el poder de los actores en la red (Ingold, 2014). Se calcula por el número de veces que un actor está en el camino de dos actores no vinculados. Estos actores que se encuentran en el camino pueden tener control sobre las interacciones entre los dos actores no adyacentes. Un actor con alto nivel de intermediación, aunque presente otras medidas de centralidad bajas, puede ejercer un rol importante en la red. A través de esta medida, se puede conocer el poder de cada actor de controlar interacciones y su potencial para acceder a recursos dentro de la red (Lin, 200I; Lienert et al., 2013), además, muestra la capacidad de un actor de servir como un intermediario o mediador entre actores o subgrupos de actores. En las redes de política, actores con alta centralidad por intermediación están mejor integrados en la red y pueden tener más influencia en el proceso de planificación y la gestión de políticas.

La centralidad por cercanía, otra medida utilizada, se enfoca en medir las distancias entre actores y compararlas con toda la red. Toma en cuenta la ubicación de un actor con respecto a otro, lo que se conoce como valor propio, y la suma de estos valores se conoce como vector propio. Una elevada centralidad por cercanía indica qué actor es más central en la red, tomando en cuenta el patrón de las distancias entre todos los actores. Esta medida identifica a los actores que tienen una ventaja posicional para acceder y compartir recursos.

Para obtener las medidas de intermediación y centralidad por cercanía, se utilizó una matriz bimodal (37 x 10) que toma en consideración el criterio individual de todos los actores entrevistados y direcciona sus criterios según la clasificación de las categorías de actores. Este análisis diferencia los resultados por fase del ciclo de política pública. De esta manera, se identificaron los actores de mayor centralidad y los cambios que presentan los actores a lo largo del ciclo.

El grado de centralidad, la otra medida utilizada, diferencia a los actores por el número de conexiones que presentan. Aquellos actores que tienen más vínculos, presentan ventajas posicionales para acceder a recursos y por lo tanto son menos dependientes de otros. También permite identificar a los actores que tienen mayor 
prestigio, a través del número de relaciones unidireccionales que reciben (centralidad por grado interno), y los actores que son más influyentes, al emitir un mayor número de relaciones hacia otros (centralidad por grado externo). Así, se identifican los actores que presentan mayores facilidades para articularse con otros (Hanneman y Riddle, 2005). En esta medida de centralidad se trabajó con la moda estadística de las categorías de actores, para obtener el valor más representativo. Se trabajó con una matriz cuadrada (Ioxio) que consiste en las nueve categorías que agrupan a los actores (tabla I) y los gabinetes de la comunidad. Se realizaron dos pruebas para obtener el grado de centralidad de los gabinetes: poniendo el mismo valor que fue otorgado por cada categoría de actores y otorgando el valor de $\odot$. En ambos casos la ubicación de este actor en la red no presenta cambios, por lo que se trabajó con el valor de o. Cada relación reconocida por los actores recibió el valor de i. En cuanto a los valores de grado interno y externo, se diferencian las relaciones unidireccionales que reciben y emiten los actores; cada una recibió el valor de I. Esto permite conocer el prestigio (grado interno) y la influencia (grado externo) de cada actor en la red. Además, se obtuvo la densidad de la red multiplicando la suma de grado de centralidad de cada fase por 100 y dividiendo este resultado para el total de interacciones posibles de una matriz cuadrada de diez actores (I0xIO-IO=90).

Para obtener el gráfico del ciclo de política pública, se sumaron las modas estadísticas de las categoría de actores de cada fase del ciclo, y de esta manera se identificó a los actores que se relacionan en mayor número de fases, siendo 5 el valor máximo y o el mínimo. Para representar gráficamente estas medidas de centralidad se utilizó NetDraw. Estos gráficos aportan a la comprensión de la estructura de la red y los patrones de participación de los actores (Molina et al., 2013).

\subsection{Panel de expertos}

Para contrastar los datos obtenidos, se identificaron instituciones que tienen experiencia en la conformación de los comussan o que desarrollan actividades en conjunto con estos a lo largo del ciclo de política pública, y que pueden ofrecer una visión objetiva del trabajo que realizan los actores en estos espacios. Estas fueron la FAO, Cáritas Nicaragua, Acción contra el Hambre, Instituto de Estudios Estratégicos y Política Pública de Nicaragua, ciussan y la Universidad Nacional de Nicaragua. De cada institución se contactó a un experto de alto nivel técnico, ${ }^{1}$ que

I Los expertos fueron identificados y contactados con apoyo de la oficina de la FAo para América Latina y el Caribe. 
trabaja directamente con los comUsSAN. Ellos identificaron a los actores de mayor importancia durante el ciclo de política pública y reflexionaron sobre los datos de grado de centralidad obtenidos en este análisis.

Cada experto de las instituciones identificadas recibió una encuesta electrónica, compuesta por cinco preguntas. Cada pregunta correspondía a una fase del ciclo de política pública, y se solicitaba identificar a los actores más relevantes en el trabajo que realizan los COMUSSAN en cada fase. En cada pregunta se utilizaron las nueve categorías de actores en estudio (tabla i) y los gabinetes de la comunidad. Cada experto podía seleccionar una o varias categorías por fase. Por cada selección que recibía una categoría se le otorgó un punto. Al ser seis los encuestados, el valor máximo que podía recibir una categoría por fase fue de seis puntos y el mínimo de cero puntos. Tomando en cuenta el número de veces que cada categoría fue seleccionada y el total de expertos consultados, se obtuvieron los porcentajes de importancia por categoría y por fase. Para obtener los resultados de importancia de las categorías en el ciclo en su conjunto, se promediaron los porcentajes de cada fase.

\section{Análisis de resultados}

Los niveles de intermediación son similares en la red del COMUSSAN (tabla 2), ya que los actores presentan constantes conexiones entre ellos durante el ciclo de política pública. En la tabla 2 se resaltan de cada fase los actores de mayor puntuación. Por parte de los actores no gubernamentales, destacan los niveles de intermediación de los gabinetes de la comunidad. Este actor es el de mayor intermediación en la red, pues dichos gabinetes tienen mayor acceso a recursos y alta injerencia en el proceso político a nivel municipal. Esto demuestra que los demás actores en la red priorizan su articulación con ellos antes que con otros actores sociales. Esto es clave para el funcionamiento del modelo de gobernanza, ya que la sociedad civil organizada tiene presencia en el ciclo. Otro actor relevante son los cooperantes. Tradicionalmente, estos actores cuentan con recursos propios, lo cual hace atractivo su vínculo con otros actores y mejora su posicionamiento en la red. En cuanto a los actores gubernamentales, el de mayor intermediación son los gobiernos locales. A estos la LSSAN les otorga la responsabilidad de coordinar las acciones del COMUSSAN, y su intermediación demuestra que tienen un alto acceso a recursos e injerencia política.

En cuanto a la centralidad por cercanía, los valores por fase son similares, lo que expresa que la cohesión de la red varía según la fase del ciclo. Como se puede observar en la tabla 2, los actores no gubernamentales de mayor centralidad 
TABLa 2. Medidas de intermediación y centralidad por cercanía de los actores que conforman el Comité Municipal de la Soberanía y Seguridad Alimentaria y Nutricional

\begin{tabular}{|c|c|c|c|c|c|c|c|c|c|c|}
\hline & \multicolumn{2}{|c|}{$\begin{array}{l}\text { AgENDA } \\
\text { PÚBLICA }\end{array}$} & \multicolumn{2}{|c|}{ FormulaCióN } & \multicolumn{2}{|c|}{ Aprobación } & \multicolumn{2}{|c|}{$\begin{array}{l}\text { IMPLE- } \\
\text { MENTACIÓN }\end{array}$} & \multicolumn{2}{|c|}{ Evaluación } \\
\hline & $\mathrm{CI}$ & $\mathrm{CC}$ & $\mathrm{CI}$ & $\mathrm{CC}$ & $\mathrm{CI}$ & $\mathrm{CC}$ & $\mathrm{CI}$ & $\mathrm{CC}$ & $\mathrm{CI}$ & $\mathrm{CC}$ \\
\hline Magfor & 0,043 & 0,268 & 0,022 & 0,273 & 0,052 & 0,357 & 0,086 & 0,286 & 0,013 & 0,198 \\
\hline MEFCCA & $0,04 \mathrm{I}$ & 0,332 & 0,064 & 0,410 & 0,009 & 0,302 & 0,050 & $0,3 \mathrm{II}$ & 0,021 & 0,267 \\
\hline Mined & 0,029 & 0,272 & 0,027 & 0,264 & $0,0 \mathrm{I} 4$ & 0,266 & 0,076 & $0,34 \mathrm{I}$ & 0,016 & 0,392 \\
\hline Minsa & 0,047 & 0,329 & 0,029 & $0,26 \mathrm{I}$ & 0,012 & 0,272 & 0,057 & 0,380 & 0,007 & 0,351 \\
\hline INTA & 0,030 & 0,299 & 0,026 & 0,324 & 0,040 & 0,278 & 0,070 & 0,278 & 0,016 & 0,386 \\
\hline Gobiernos locales & $0, I 47$ & 0,459 & 0,078 & 0,436 & $0, I I I$ & 0,538 & 0,067 & 0,369 & 0,023 & 0,303 \\
\hline CIUSSAN & 0,028 & 0,298 & $0,04 \mathrm{I}$ & 0,205 & 0,017 & 0,208 & 0,027 & 0,163 & 0,013 & 0,284 \\
\hline Cooperantes & 0,083 & 0,286 & 0,159 & 0,388 & 0,052 & 0,217 & 0,134 & 0,420 & 0,078 & 0,454 \\
\hline $\begin{array}{l}\text { Asociaciones de } \\
\text { productores }\end{array}$ & 0,006 & 0,096 & 0,001 & 0,034 & 0,014 & $0, \mathrm{IIO}$ & 0,003 & 0,099 & - & 0,044 \\
\hline $\begin{array}{l}\text { Gabinetes de la } \\
\text { comunidad }\end{array}$ & 0,118 & 0,390 & 0,032 & 0,359 & 0,059 & 0,406 & 0,068 & $0,36 \mathrm{I}$ & 0,034 & 0,284 \\
\hline
\end{tabular}

$\mathrm{CI}=$ centralidad por intermediación

$\mathrm{CC}=$ centralidad por cercanía

FUENTE: elaboración propia

por cercanía en las fases de agenda pública, formulación y aprobación son los gabinetes de la comunidad, siendo los más centrales en la red. Esto responde al nivel territorial de trabajo y muestra la importancia que tiene la organización social en los procesos municipales de toma de decisión. Los cooperantes tienen elevada centralidad por cercanía durante el ciclo, en especial en las fases de implementación y evaluación, lo que responde al trabajo con recursos propios e iniciativas puntuales de seguimiento y evaluación que realizan este tipo de instituciones. Los actores gubernamentales son los de mayor centralidad, entre estos destacan los gobiernos locales en las tres primeras fases del ciclo de política, respondiendo así a los intereses del territorio y a un proceso de descentralización de la política pública.

Si se comparan las figuras i y 2, se puede diferenciar la variación que tiene la centralidad por cercanía de las categorías de actores en las fases de agenda pública e implementación. En la primera, las categorías de actores tienen menor centralidad por cercanía, por eso los tamaños de los nodos que los representan son menores. En comparación con la figura 2, se puede observar un ligero aumento de tamaño en gobiernos locales y gabinetes de la comunidad. Además, se observa que las categorías de actores dejan un vacío central en la red y que se conforman subgrupos, por la mayor interdependencia que existe entre estos. El primer subgrupo prioriza su accionar con los gobiernos locales, en este participan 
Magfor e INTA, actores vinculados al sector productivo agropecuario, lo que podría direccionar los esfuerzos únicamente hacia este sector. En el segundo grupo se priorizan las acciones con los gabinetes de la comunidad, y su trabajo, según los actores participantes, se direcciona hacia educación y salud. Este subgrupo trabaja directamente con la sociedad civil, dejando de lado a la autoridad política local (gobiernos locales) en la priorización de políticas. En el tercer grupo se encuentran la Academia, los cooperantes que poseen recursos propios y el Mefcca. Esto demuestra que existen diferencias en la cohesión de ciertos actores en esta fase.

En la figura 2 se presenta la centralidad por cercanía de los actores del COMUSSAN en la fase de implementación. Se observa que el tamaño de los nodos aumenta en comparación con la figura I, y se destacan entre estos los gobiernos locales, los cooperantes y el Minsa. Las diferencias de centralidad de los actores son mínimas, lo cual demuestra que se encuentran de forma más centralizada, con una mayor cohesión de la red en esta fase. CIUSSAN y la Asociación de Productores se alejan del centro de la red, lo que demuestra que son los de menor articulación.

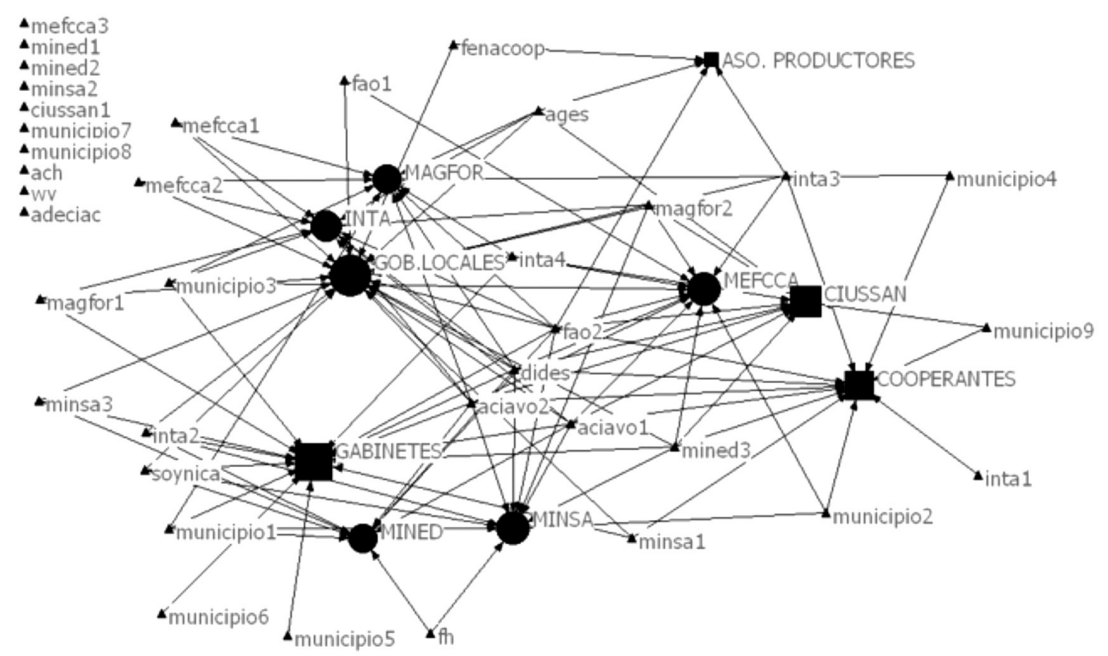

Figura 1. Centralidad por cercanía de la red de actores que participan en el Comité Municipal de la Soberanía y Seguridad Alimentaria y Nutricional y que hacen parte de la fase de agenda pública

Nota: la matriz utilizada para graficar es 37 x ı. Las categorías de actores están representadas por nodos cuadrados las no gubernamentales y nodos circulares las gubernamentales, y los 37 actores que participaron por nodos triangulares. El tamaño de los nodos de las categorías varía según su centralidad. Los nodos que están en el costado superior izquierdo representan a los actores que están desconectados de la red en las fases en estudio.

FuEnTE: elaboración propia 


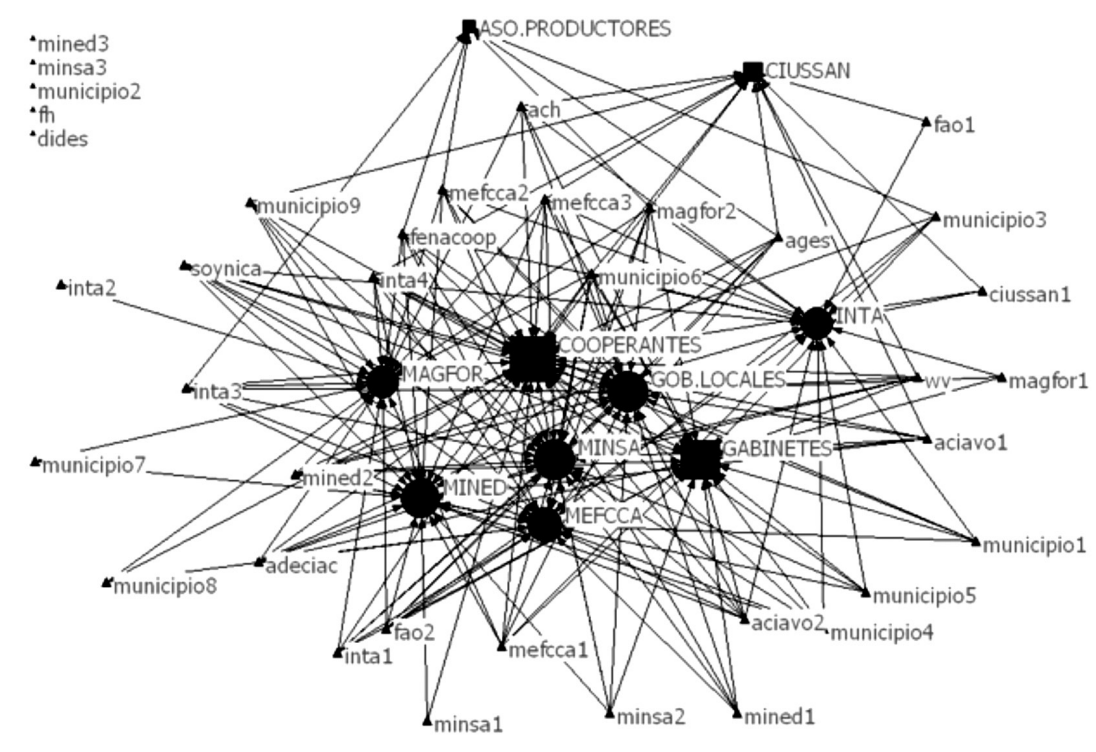

Figura 2. Centralidad por cercanía de la red de actores que participan en el Comité Municipal de la Soberanía y Seguridad Alimentaria y Nutricional en la fase de implementación

Nota: la matriz utilizada para graficar es 37 x ı. Las categorías de actores están representadas por nodos cuadrados las no gubernamentales y nodos circulares las gubernamentales, y los 37 actores que participaron por nodos triangulares. El tamaño de los nodos de las categorías varía según la centralidad que posean. Los nodos que están en el costado superior izquierdo representan a los actores que están desconectados de la red en las fases en estudio.

Fuente: elaboración propia

En cuanto al grado de centralidad (tabla 3), los actores no gubernamentales que tienen elevada interacción en la red son los cooperantes, los gabinetes de la comunidad y las asociaciones de productores; estos presentan niveles similares en las fases de agenda pública, formulación e implementación, lo cual muestra que existe involucramiento de la sociedad civil y organizaciones no gubernamentales en la puesta en marcha de las políticas a nivel municipal. El ciussan es el actor que menor interacción tiene a lo largo del ciclo de política pública, a pesar de reunir a dieciséis universidades que han incorporado a la SAN como eje transversal en su currículo académico, ser reconocido como un actor que genera espacios de discusión e investigación sobre la sAN, y apoyar la formación de técnicos locales en planificación e implementación de la LSSAN; la red del comUSSAN no aprovecha su potencial. Es necesario que se definan los roles que debe cumplir este actor en la puesta en marcha de políticas públicas. De los actores gubernamentales destaca el Magfor, como el actor que mayor interacción genera a lo largo del ciclo. Este actor 
es el de mayor influencia en la red. En la fase de implementación todos los actores tienen elevada participación, lo que concuerda con las otras medidas de centralidad y demuestra que existe una mayor articulación entre los actores al momento de implementar acciones a nivel municipal.

TABLA 3. Grado de centralidad y densidad de la red de los actores que conforman el Comité Municipal de la Soberanía y Seguridad Alimentaria y Nutricional, por fase del ciclo de política pública. Incluye el porcentaje de importancia de cada actor otorgado por los expertos consultados

\begin{tabular}{|c|c|c|c|c|c|c|c|c|c|c|c|c|c|c|c|c|}
\hline & \multicolumn{3}{|c|}{$\begin{array}{l}\text { Agenda } \\
\text { PúBLICA }\end{array}$} & \multicolumn{3}{|c|}{ Formulación } & \multicolumn{3}{|c|}{ Aprobación } & \multicolumn{3}{|c|}{$\begin{array}{l}\text { IMPLE- } \\
\text { MENTACIÓN }\end{array}$} & \multicolumn{3}{|c|}{ Evaluación } & \multirow{2}{*}{$\begin{array}{c}\text { OPINIÓN } \\
\text { DE EXPERTOS } \\
\text { Importancia } \\
(\%) \\
\end{array}$} \\
\hline & $\mathrm{GdC}$ & GE & GI & $\mathrm{GdC}$ & $\mathrm{GE}$ & GI & $\mathrm{GdC}$ & $\mathrm{GE}$ & GI & $\mathrm{GdC}$ & $\mathrm{GE}$ & GI & $\mathrm{GdC}$ & $\mathrm{GE}$ & GI & \\
\hline Magfor & 6 & 6 & I & 6 & 4 & 2 & 6 & 6 & 2 & 8 & 8 & 3 & $\circ$ & $\circ$ & $\circ$ & $65,7 I$ \\
\hline MEFCCA & 4 & 3 & 3 & 3 & $\circ$ & 3 & 2 & 2 & I & 8 & 7 & 5 & I & $\circ$ & I & 42,86 \\
\hline Mined & 2 & $\circ$ & 2 & I & $\circ$ & I & $\circ$ & $\circ$ & $\circ$ & 7 & 5 & 5 & $\circ$ & $\circ$ & $\circ$ & $45,7 \mathrm{I}$ \\
\hline Minsa & 5 & 2 & 3 & 2 & $\circ$ & 2 & I & $\circ$ & I & 6 & 2 & 5 & $\circ$ & $\circ$ & $\circ$ & $45,7 \mathrm{I}$ \\
\hline INTA & 6 & 4 & 3 & 3 & 2 & I & 5 & 4 & 2 & 9 & 7 & 6 & I & I & $\circ$ & 34,29 \\
\hline $\begin{array}{l}\text { Gobiernos } \\
\text { locales }\end{array}$ & 6 & $\circ$ & 6 & 2 & $\circ$ & 2 & 3 & $\circ$ & 3 & 7 & 5 & 6 & I & 0 & I & $97, I 4$ \\
\hline CIUSSAN & 2 & $\circ$ & 2 & 3 & 3 & 0 & I & $\circ$ & I & 3 & 2 & 2 & I & I & $\circ$ & 34,29 \\
\hline Cooperantes & 5 & 4 & I & 5 & I & 4 & I & $\circ$ & I & 9 & 4 & 8 & I & $\circ$ & I & 48,57 \\
\hline $\begin{array}{l}\text { Asociaciones } \\
\text { de } \\
\text { productores }\end{array}$ & 6 & 6 & 0 & 6 & 6 & 0 & I & I & 0 & 8 & 8 & I & I & I & 0 & 60,00 \\
\hline $\begin{array}{l}\text { Gabinetes de } \\
\text { la comunidad }\end{array}$ & 4 & $\circ$ & 4 & I & $\circ$ & I & 2 & 0 & 2 & 7 & 0 & 7 & $\circ$ & 0 & 0 & 37,14 \\
\hline $\begin{array}{l}\text { Total de } \\
\text { relaciones }\end{array}$ & 46 & & & 32 & & & 22 & & & 72 & & & 6 & & & \\
\hline $\begin{array}{l}\text { Densidad de } \\
\text { la red }(\%)\end{array}$ & $5 \mathrm{I}, \mathrm{II}$ & & & 35,56 & & & 24,44 & & & 80,00 & & & 6,67 & & & \\
\hline
\end{tabular}

$\mathrm{GDC}_{\mathrm{D}}=$ grado de centralidad

$\mathrm{GE}=$ grado externo de centralidad

$\mathrm{GI}=$ grado interno de centalidad

FuENTE: elaboración propia 
La tabla 3 presenta los valores de grado externo y grado interno, diferenciando las relaciones unidireccionales que emiten o reciben los actores para conocer su influencia y prestigio. Esto demuestra que los actores Magfor, asociaciones de productores, gobiernos locales y cooperantes son los que mayor número de vínculos generan en la red; los dos primeros como emisores (mayor influencia), los dos últimos como receptores (mayor prestigio). Por esta condición, estos actores tienen mayor acceso a recursos y no presentan dependencia de ningún actor para ejecutar sus iniciativas o poner en marcha las fases del ciclo de política pública.

En la última fila de la tabla 3 se presentan los resultados de la densidad de red. Estos demuestran que la etapa de implementación genera el $80 \%$ de interacciones posibles, y conjuntamente con la fase de agenda pública con el 5I,II \%, son las de mayor interacción en el ciclo de política pública. Las fases de formulación y aprobación tienen menor grado de interacción, con el 35,56 y 24,44\%, respectivamente. Si esta participación se concentra de manera adecuada, puede aportar a un mayor dinamismo del ciclo. La fase de evaluación genera baja interacción, las iniciativas que se están llevando a cabo responden a actividades puntuales que involucran a otros actores de la red. La densidad de las interacciones de la red aumenta según el número de actores que se involucren, pero existen límites prácticos sobre el número de conexiones o vínculos que puede manejar un actor. La figura 3 demuestra que no todos los actores tienen capacidad para participar en todo el ciclo.

A los expertos se les consultó sobre qué actor, según las categorías establecidas, tiene más relevancia en cada fase del ciclo de política pública. Después de su respuesta, se les presentaron los resultados del grado de centralidad a lo largo del ciclo, obtenido a través de las encuestas, y posteriormente se abrió un espacio para comentarios. La opinión de los expertos reconoce que los gobiernos locales son el actor de mayor importancia durante el ciclo de política pública, lo que concuerda con los altos niveles de centralidad que se presentan en las tablas 2 y 3 . El Magfor con un porcentaje de importancia de 65,71\% y los gabinetes de la comunidad con el 60\% son actores importantes en el comussan, según los expertos. En contraste con los datos de las medidas de centralidad, el Magfor es reconocido como el actor que más interacciones genera en la red y los gabinetes de la comunidad tienen niveles altos de centralidad en varias fases del ciclo, como se observa en la tabla 2. A continuación se identifican dos grupos con menor importancia, el primero conformado por Mefcca, Mined, Minsa y los cooperantes, a quienes se les otorgaron porcentajes entre 42 y $49 \%$. Estos actores muestran niveles de intermediación y centralidad por cercanía 
similares. En cuanto a grado de centralidad, se diferencian los cooperantes, lo que también reconoce el grupo de expertos (tabla 3). El segundo grupo está compuesto por INTA, CiUssan y la Asociación de Productores, estos dos últimos son los que menor intermediación y centralidad por cercanía poseen. Por su parte, el INTA, a pesar de presentar un bajo porcentaje de importancia, tiene un elevado grado externo de centralidad (tabla 3), lo que demuestra que no tiene elevado prestigio, pero puede influir en el accionar de la red.

En los comentarios a los resultados se menciona que las instituciones que tienen mayor importancia son las de mayor presencia en los municipios, lo que genera más confianza a otros actores para trabajar con ellos. Se reconoce que los gobiernos locales son el actor más relevante, porque la ley les otorga las principales responsabilidades dentro del comussan, y por el esfuerzo de descentralización que se lleva a cabo en el país, son los principales promotores de la sAN en sus localidades. Se afirma asimismo que los gabinetes de la comunidad consideran a estas instancias municipales un espacio de disputa política, por lo que participan constantemente. En este sentido, las disputas políticas en el interior de cada municipalidad condicionan el tipo y la intensidad de la participación en el comussan. Si los actores externos no se ajustan a las normas de participación establecidas por el COMUSSAN, se dificulta su articulación en estos espacios. Además, se manifiesta que los actores vinculados al sector agropecuario tienen mayor participación a lo largo del ciclo, y otros actores sectoriales como Mined y Minsa cumplen roles claves en la generación de políticas públicas intersectoriales, a pesar de la menor participación que presentan. Los expertos consideran que la Universidad puede desempeñar un papel importante en el escenario político que se presenta, a través de la generación de espacios de debate ampliado para lograr consensos graduales.

La figura 3 muestra el grado de centralidad de las categorías de actores del comussan a lo largo del ciclo de política pública. Se observa que los actores con mayor centralidad son Magfor e INTA; estos se convierten en actores relevantes al momento de articular a los demás actores de la red. Por su parte, el ciussan se presenta como el actor con menor articulación. Estos resultados muestran que los actores interactúan con mayor frecuencia en una fase del ciclo y que interactuar en dos o más fases presenta mayores dificultades, por lo cual las relaciones disminuyen. Esto manifiesta que dentro de estos espacios de articulación interinstitucional no todos los participantes tienen las mismas responsabilidades y que su participación no se da en condiciones de igualdad. Los actores que reconocen participar en todas las fases del ciclo son los gobiernos locales y las asociaciones de productores, quienes presentan 


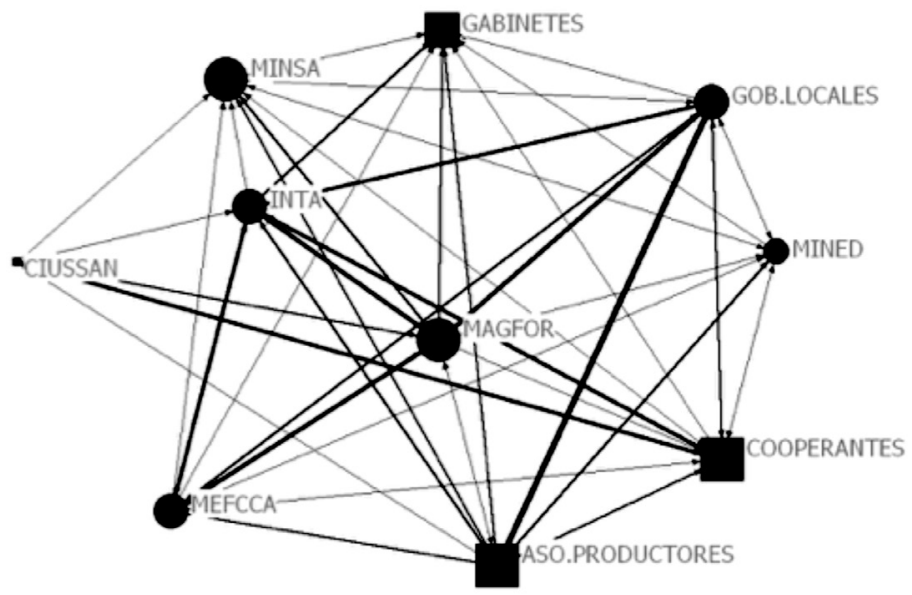

Figura 3. Grado de centralidad de los actores del Comité Municipal de la Soberanía y Seguridad Alimentaria y Nutricional durante el ciclo de política pública

NoтA: para diferenciar los actores de la red, los actores gubernamentales se representaron con nodos circulares y los actores no gubernamentales con nodos cuadrangulares. Las conexiones entre actores están representadas por líneas que aumentan su grosor según el número de fases en las que trabajen dos actores en conjunto.

FUENTE: elaboración propia

conexiones fuertes y tienen mayor cohesión en la red. El resultado de estos acuerdos identifica a los actores gubernamentales, en especial al Magfor a nivel nacional y los gobiernos locales a nivel municipal, como los principales, al promover la mayor articulación de la red de actores en la promoción de políticas públicas.

\section{Discusión}

La LSSAN propone que el Estado sea el principal actor en garantizar la seguridad alimentaria y busca cumplirlo a través del modelo de gobernanza que se está implementando. Pierre y Peters (2005) sostienen que las redes de actores que mejor conectadas estén con los actores del sector público tienen mayor capacidad de incidencia sobre las políticas públicas. En este modelo, a pesar de que las conexiones entre actores gubernamentales y no gubernamentales son similares a lo largo del ciclo de política, estos últimos actores tienen baja incidencia en las fases de formulación y aprobación. El involucramiento de los actores no gubernamentales en el COMUSSAN es un componente importante en la legitimación del accionar público, pero debe trascender su rol si esperamos que esta red de actores municipales 
aumente su cohesión social y cumpla con las funciones otorgadas por la LSSAN. Para esto es necesario que los actores gubernamentales muestren flexibilidad en la toma de decisiones y hagan concesiones a lo largo del ciclo de política, otorgando responsabilidades y protagonismo a la participación social.

La red COMUSSAN reconoce que ningún actor a nivel municipal posee los recursos y la capacidad para abordar la lucha contra el hambre de manera unilateral, lo cual requiere coordinar esfuerzos bajo objetivos comunes. Como mencionan estudios similares (Grimes, 2013; Duncan y Barling, 2012; Bernauer y Gampfer, 2013; Blair, 2000) y reconocen otros investigadores (Kickert et al., ı997; Duit y Galaz, 2008), lo cual tipo de redes conformadas por intereses comunes se convierten en instancias de mediación e influencia en el nivel territorial, lo que no solo permite llegar a consensos en la implementación de políticas públicas más cercanas a las necesidades de los territorios, sino que además marca el accionar de otros actores que estén fuera de la red.

Los actores que mayor importancia tienen en la red de actores COMUSSAN son los gobiernos locales, lo cual fortalece la descentralización en la generación de políticas públicas. Estos actores, que según la LSSAN son los encargados de dirigir las actividades a nivel municipal, presentan apertura a lo largo del ciclo de política pública. Su prestigio (grado interno) es elevado, al ser el actor que mayor número de relaciones unidireccionales recibe en la priorización de las políticas públicas. La limitación de recursos técnicos y económicos con que cuentan es uno de los factores que han influido el trabajo de los COMUSSAN, especialmente en la articulación interinstitucional.

Los gobiernos locales tienen una centralidad por cercanía similar a lo largo del ciclo, situando a estos actores de forma central en la red, lo que favorece su trabajo por los canales cortos de intermediación con otros actores (Wasserman y Faust, I994).

Sus niveles de intermediación no son elevados en las fases de formulación e implementación, y ello demuestra que los actores con mayores capacidades toman un papel más relevante en la red.

De la participación del sector público resalta el grado de centralidad de Magfor, este presenta alta interacción en las cuatro primeras fases del ciclo y es el actor que más interacciones genera en toda la red. Su intermediación sobresale en la fase de agenda pública, aprobación e implementación, lo que demuestra que a pesar de no estar relacionado con todos actores de forma directa, su rol es clave para que los actores trabajen en conjunto. Al ser el actor que más dinamiza la red, demuestra que las iniciativas sectoriales agropecuarias son las de mayor importancia en el COMUSSAN. El Magfor, al generar mayor número de conexiones, debe ser capaz 
de articularse con los demás actores, a pesar de las diferentes percepciones sobre problemas y soluciones que puedan presentar, como mencionan estudios similares (Kickert et al., I997; Giest y Howlett, 20I4; Capaldo, 20I4; Candel, 20I4), procurando de esta manera un adecuado funcionamiento de la red. Las medidas de centralidad utilizadas reflejan una mayor capacidad de los actores gubernamentales de conducir procesos de priorización y de toma de decisiones, ejerciendo así un mayor control de las políticas públicas.

En cuanto a los actores no gubernamentales, los cooperantes son los de mayor relevancia. Estos tienen alto grado de centralidad y reciben un mayor número de relaciones unidireccionales. El poseer recursos propios para implementar acciones es uno de los factores que promueven su centralidad en la red a nivel municipal. En este aspecto el COMUSSAN se convierte en una instancia crucial para el diálogo y el direccionamiento de esfuerzos por parte de las instituciones participantes y futuros actores que se incorporen. Además, es una instancia clave para evitar la duplicidad de acciones y promover un mayor ordenamiento territorial de las iniciativas y el impacto que se genera. Los otros actores no gubernamentales deberán incorporarse en mayor medida a las acciones de la red, lo que favorecerá la transparencia del modelo de gobernanza (Blair, 2000; Grimes, 20I3; Ingold, 20I4; Flores et al., 2009). En un estudio similar realizado por Flores y Gómez-Sánchez (2010), se establece que la eficacia de este tipo de espacios de participación social está determinada por los niveles de involucramiento de la sociedad civil y por la injerencia que puedan tener en las políticas públicas; este factor se debe tomar en cuenta para buscar una mayor participación de los actores sociales en los espacios locales de articulación. Los gabinetes de la comunidad son claves en el adecuado funcionamiento del COMUSSAN, estas estructuras sociales organizadas permiten un mayor acercamiento a los problemas locales y fomentan la construcción conjunta de iniciativas para darles solución. El prestigio que tienen es elevado a lo largo del ciclo, especialmente en las fases de priorización e implementación, lo que favorece el seguimiento y veeduría social de las políticas que se lleven a cabo; además, su cercanía en la red es elevada, lo cual favorece una comunicación y un intercambio de recursos muy dinámicos.

En este modelo de gobernanza, los actores públicos mantienen una posición central en el ciclo de política. Los actores sociales aportan a la puesta en marcha de políticas públicas, en especial en la fase de priorización, pero la toma de decisiones la realizan principalmente los actores públicos. La fase de implementación de este modelo puede ser descrita como un proceso interactivo entre todos los actores de la red. La ejecución de políticas frecuentemente lleva de forma inherente procesos de 
negociación entre actores públicos y los intereses sociales, en donde el COMUSSAN desempeña un papel importante para fijar objetivos comunes y direccionar las políticas públicas a nivel municipal, como lo menciona Godek (20I4). Este modelo de gobernanza fomenta una mayor articulación horizontal a nivel municipal en las fases de priorización e implementación de políticas públicas (las de mayor densidad de red), en tanto que en las de formulación y aprobación disminuye la articulación de actores a nivel municipal y hay mayor influencia de interacciones verticales, lo cual influye en las acciones del COMUSSAN desde instancias a nivel nacional. En la fase de evaluación las interacciones que existen son limitadas, y ello disminuye la capacidad de incidir por parte de los actores no gubernamentales, a través del control social que pueden realizar a la construcción y ejecución de las políticas públicas (Blair, 2000).

\section{Conclusiones}

Con estos resultados se entiende de mejor manera el funcionamiento de las instancias de articulación interinstitucional que se han llegado a implementar a partir de lo propuesto por la LSSAN en Nicaragua. Aunque los niveles de articulación podrían ser mejores, lo que han conseguido los actores que participan en el COMUSSAN ilustra sobre cómo se debe replicar esta iniciativa en otros municipios y qué factores se debe tomar en cuenta al momento de conformar las instancias distritales y nacionales. Este análisis de redes políticas posibilita a los tomadores de decisión gubernamentales y no gubernamentales ser críticos sobre el accionar que tienen y les permite discutir sobre la manera más adecuada de mejorar o iniciar su participación en este tipo de instancias.

En particular, el análisis de la red de actores que participan en el COMUSSAN muestra que existe una relación inversa entre la inclusión de un actor en la red y la autonomía que pueda presentar en sus acciones, según lo expresan los expertos y por los datos que presentan las fases de priorización y aprobación, donde existe dependencia de las decisiones a nivel nacional. Esta característica del modelo de gobernanza dificulta la participación de actores que no concuerdan con los procesos que se promueven desde el nivel nacional. El aporte de otros actores a lo largo del ciclo de política pública se ve limitado por la dificultad de mantener la autonomía en su participación.

Para que este modelo de gobernanza tenga mayor impacto, es necesario institucionalizar estos espacios de articulación municipales. Ciertos municipios han aprobado ordenanzas municipales, lo que respaldará su funcionamiento, a pesar de la tendencia política de turno. Los COMUSSAN deben presentar mayor apertura a la inclusión de otros actores, para el reconocimiento, la reflexión y la 
discusión del problema del hambre. Es muy importante que en estos espacios se establezcan objetivos y metas comunes a corto, mediano y largo plazo que aseguren un trabajo articulado y una coordinación adecuada de su accionar. En este sentido, la transparencia debe ser una condición básica entre los actores involucrados y los beneficiarios. Se debe promover asimismo una retroalimentación continua para ajustar de la mejor manera las políticas públicas a los contextos locales, y es necesario que haya un constante monitoreo y evaluación para medir progresos, presentar avances a la sociedad y redireccionar el accionar, si ello es preciso.

Los resultados obtenidos en esta investigación permiten identificar pautas de importancia que pueden ser tomadas en cuenta para efectivizar iniciativas similares; estas son: I) los gobiernos municipales deben ser los principales actores en la gestión de las políticas locales y en promover la participación social en sus territorios. Esto no solo legitimará su actuación, sino que además permitirá que los actores gubernamentales y los no gubernamentales sumen recursos para alcanzar objetivos comunes; 2) es necesario promover una participación plural, representativa e inclusiva en los espacios locales de articulación, y que todos los actores puedan actuar en condiciones similares. Ello evitará que estos espacios respondan a intereses políticos; y 3) la sociedad civil desempeña un rol primordial en el control y la veeduría social de las políticas públicas, y a través de espacios como el comussan puede encontrar una plataforma de actores que le otorgue soporte técnico y político para cumplir con esta responsabilidad.

Este tipo de análisis de redes de políticas permite identificar de qué manera se está promoviendo la gobernanza en el combate al hambre. $\mathrm{Al}$ ser varios los países que implementan nuevos marcos legales y nuevas estructuras interinstitucionales en América Latina, el uso de este análisis ayuda al desarrollo de las nuevas propuestas de articulación entre el Estado y la sociedad civil, así como a buscar características similares y condiciones que hayan influido en el funcionamiento de instancias interinstitucionales en diferentes países de la región.

\section{Agradecimientos}

Los autores expresan su agradecimiento a Alberto Ramírez Fiora y Ricardo Rapallo, de la Oficina Regional de la FAO para América Latina, y a todo el personal de la Oficina de la FAO en Nicaragua, por su apoyo en esta investigación. Una versión previa de esta investigación fue presentada en el Congreso Internacional "Anhelando un futuro sin despilfarro ni pobreza alimentaria: retos sociales", en Bilbao, España en noviembre del 2015. 


\section{Referencias}

Aguilar, L. F. (2006). El aporte de la política pública y de la nueva gestión pública a la gobernanza. Revista del CLAD, Reforma y Democracia, 39, I-I5.

Bernauer, T. y Gampfer, R. (2013). Effects of civil society involvement on popular legitimacy of global environmental governance. Global Environmental Change, 23(2), 439-449.

Blair, H. (2000). Participation and accountability at the periphery: democratic local governance in six countries. World Development, 28(I), 2I-39.

Borgatti, S. P., Everett, M. G. y Freeman, L. C. (2002). Ucinet for Windows: Software for social network analysis.

Candel, J. J. (20I4). Food security governance: a systematic literature review. Food Security, 6(4), 585-60I.

Capaldo, A. (2014). Network governance: A cross-level study of social mechanisms, knowledge benefits, and strategic outcomes in joint-design alliances. Industrial Marketing Management, 43(4), 685-703.

Cofre, E., Yagüe, J. L. y Moncayo, M. (2015). Seguridad y soberanía alimentaria: análisis comparativo de las leyes en siete países de América Latina. Revista Española de Estudios Agrosociales y Pesqueros, 242, 49-87.

Duit, A. y Galaz, V. (2008). Governance and complexity-emerging issues for governance theory. Governance, 2I(3), 3II-335.

Duncan, J. y Barling, D. (20I2). Renewal through participation in global food security governance: implementing the international food security and nutrition civil society mechanism to the committee on world food security. International Journal of the Sociology of Agriculture and Food, I9(2), I43-I6I.

Fischer, M. (20II). Social network analysis and qualitative comparative analysis: Their mutual benefit for the explanation of policy network structures. Methodological Innovations Online, 6(2), 27-5I.

Flores, W., Ruano, A. y Funchal, D. (2009). Social participation within a context of political violence: implications for the promotion and exercise of the right to health in Guatemala. Health and Human Rights, 37-48.

Flores, W. y Gómez-Sánchez, I. (2010). La gobernanza en los Consejos Municipales de Desarrollo de Guatemala: Análisis de actores y relaciones de poder. Revista de Salud Pública, 12, 138-150.

Food and Agriculture Organization (FAO) (1996). Rome Declaration on world food security and world food summit plan of action. Roma: FAO. 
Food and Agriculture Organization (FAO) (2015). The state of food insecurity in the world. Roma: FAO.

Fontana, A. y Yagüe, J. L. (20I2). Conceptualizing governance in Latin America. En A. Cazorla y J. Midgley, Planning and community development: Case studies. Madrid: uc Berkeley - Technical University of Madrid.

Giest, S. y Howlett, M. (2014). Understanding the pre-conditions of commons governance: The role of network management. Environmental Science \& Policy, $36,37-47$.

Godek, W. (2014). The institutionalization of food sovereignty: the case of Nicaragua's law of food and nutritional sovereignty and security (Doctoral dissertation). Rutgers University, Graduate School, Newark.

Grimes, M. (2013). The contingencies of societal accountability: Examining the link between civil society and good government. Studies in Comparative International Development, 48 (4), 380-402.

Grote, U. (20I4). Can we improve global food security? A socio-economic and political perspective. Food Security, 6 (2), 187-200.

Hanneman, R. y Riddle, M. (2005). Introduction to social network methods. Riverside, CA: University of California, Riverside.

Ingold, K. (20I4). How involved are they really? A comparative network analysis of the institutional drivers of local actor inclusion. Land Use Policy, 39, 376-387.

Jann, W. y Wegrich, K. (2007). 4 theories of the policy cycle. Handbook of Public Policy Analysis, 43 .

Kickert, W. J., Klijn, E. H. y Koppenjan, J. F. M. (Eds.) (1997). Managing complex networks: strategies for the public sector. Sage.

Kooiman, J. (2003), Governing as governance. Londres: Sage.

Kooiman, J. (Ed.). (1993) Modern governance: new government-society interactions. Sage.

Lienert, J., Schnetzer, F. e Ingold, K. (2013). Stakeholder analysis combined with social network analysis provides fine-grained insights into water infrastructure planning processes. Journal of Environmental Management, 125, 134-I48.

Lin, N. (200I). Social capital. Cambridge: Cambridge University Press.

Molina, J. L., Maya-Jariego, I. y McCarthy, C. (2013). Giving meaning to social networks: Methodology for conducting and analyzing interviews based on personal network visualizations. En Mixing methods in social network research. Cambridge University Press. 
Paarlberg, R. L. (2002). Governance and food security in an age of globalization (vol. 36). Intl Food Policy Res Inst. Recuperado de http://www.oda-alc.org/ documentos/1307636457.pdf

Pérez-Escamilla, R. (2012). Can experience-based household food security scales help improve food security governance? Global Food Security, I(2), I20-I25.

Pierre, J. y Peters, G. B. (2005). Governing complex societies: Trajectories and scenarios. Basingstoke: Palgrave MacMillan.

Rhodes, R. A. W. (I996). The new governance: governing without governmentI. Political Studies, 44(4), 652-667.

Rhodes, R. A. (2007). Understanding governance: Ten years on. Organization Studies, 28(8), I243-I264.

Rodríguez, J. A. (2005). Análisis estructural y de redes. Centro de Investigaciones Sociológicas (CIS).

Stoker, G. (I998). Governance as theory: five propositions. International Social Science Journal, $50(155)$, I7-28.

Wasserman, S. y Faust, K. (1994). Social network analysis: Methods and applications (vol. 8). Cambridge University Press. 